\title{
Distribución geográfica de especies de Leishmania aisladas de pacientes consultantes al Instituto Nacional de Dermatología Federico Lleras Acosta, E.S.E., 1995-2005
}

Clemencia Elena Ovalle, Luisa Porras, Maritza Rey, Melania Ríos, Yenny Carolina Camargo

Centro Dermatológico Federico Lleras Acosta, E.S.E., Bogotá, D. C., Colombia.

Introducción. El mapa de la distribución de especies de Leishmania en Colombia no se ha actualizado desde hace siete años.

Objetivo. Describir la distribución de las especies de Leishmania a partir de los aislamientos de pacientes consultantes al Instituto Nacional de Dermatología durante el período 1995 a 2005

Materiales y métodos. Se realizó un estudio descriptivo de distribución geográfica de las especies de Leishmania, a partir de los aislamientos obtenidos de 137 pacientes consultantes al Instituto Nacional de Dermatología Federico Lleras Acosta E. S. E, con diagnóstico confirmado de leishmaniasis. Se revisó la historia clínica obteniendo la información de forma clínica, sitio probable de infección, edad y género. Se tomaron y cultivaron muestras de las lesiones y los aislamientos se tipificaron por anticuerpos monoclonales, comparando el $10 \%$ de los resultados con los obtenidos por reacción en cadena de la polimerasa (PCR) y patrones isoenzimáticos. Resultados. La frecuencia encontrada de los 137 aislamientos fue: Leishmania panamensis 74,45\%; Leishmania braziliensis 15,33\%; Leishmania guyanensis 0,73\%; Complejo Leishmania mexicana $3,65 \%$, Leishmania mexicana $5,11 \%$ y el $0,73 \%$ restante correspondió a un aislamiento que no se pudo caracterizar por anticuerpos monoclonales. La distribución de $L$. braziliensis, L. panamensis y L. guyanensis fue concordante con lo reportado en estudios anteriores pero para las especies del complejo L. mexicana se encontraron procedencias no reportadas previamente: Caldas, Santander, Cundinamarca, Caquetá, Casanare, Cauca y Valle del Cauca.

Conclusión. El complejo L. mexicana presenta una distribución más amplia de la reportada previamente. La utilidad de los monoclonales especie-específicos, los patrones isoenzimáticos y la PCR para L. mexicana y L. amazonensis fue limitada.

Palabras clave: leishmaniasis cutánea, epidemiología, clasificación, anticuerpos monoclonales, Leishmania

Geographic distribution of Leishmania species isolated from patients at the National Institute of Dermatology Federico Lleras Acosta E.S.E., 1995-2005

Introduction. The Colombian distribution map of Leishmania species has not been updated since seven years ago.

Objective. To describe the distribution of Leishmania species isolated from patients attended at the National Institute of Dermatology during the period 1995 to 2005.

Materials and methods. A descriptive study of the geographic distribution of Leishmania species was made from 137 isolates obtained from patients consulting the National Institute of Dermatology "Federico Lleras Acosta E.S.E", with confirmed diagnosis of leishmaniasis. The clinical history was reviewed to obtain information on the clinical presentation, most probable place of infection, age and gender. Samples were taken and cultured and the isolates were typed by monoclonal antibodies, comparing $10 \%$ of the results with those obtained by PCR and isoenzymatic patterns.

Results. L. panamensis accounted for $74.45 \%$ of the 137 isolates studied, L. braziliensis for $15.33 \%$, L. guyanensis for $0.73 \%$; L. mexicana complex for $3.65 \%$, L. mexicana $5.11 \%$ and the 
remaining $0.73 \%$ corresponded to an isolate which could not be characterized by monoclonal antibodies. The distribution of L. braziliensis, L. panamensis and L. guyanensis was similar to that reported in previous studies but species of the $L$. mexicana complex, were found in patients from the departments of Caldas, Santander, Cundinamarca, Caquetá, Casanare, Cauca and Valle del Cauca, where they had not been previously reported.

Conclusion. Species of the L. mexicana complex display a wider distribution than previously reported. The usefulness of species-specific monoclonal antibodies, isoenzymatic patterns, and PCR for identification of L. mexicana and L. amazonensis was limited.

Key words: cutaneous leishmaniasis, epidemiology, classification, monoclonal antibodies, Leishmania

La epidemiología de la leishmaniasis se puede alterar por cambios en algún punto de la triada epidemiológica (humanos, reservorios y flebótomos). Los factores ambientales, el desarrollo económico, el aumento en la urbanización, la deforestación, la migración de áreas rurales a urbanas con creación de nuevas zonas de asentamiento humano y el conflicto armado pueden ser responsables de la dispersión del vector, lo cual hace que la epidemiología de la enfermedad cambie y, por consiguiente, la distribución de las cepas de Leishmania $(1,2)$.

La historia taxonómica de Leishmania inicia con la descripción del parásito y la definición del género hecha por Ross en 1903, seguido de la reagrupación del genero Leishmania en los subgéneros Viannia y Leishmania por Lainson y Shaw en 1987, basados en el desarrollo del parásito en el intestino de los vectores; por ello, inicialmente los criterios de identificación y clasificación del parásito se basaban en las características extrínsecas como la biología, las manifestaciones clínicas, los aspectos geográficos y epidemiológicos que ofrecían un acercamiento a la especie infecciosa (3). En 1998, Cupolillo et al. agrupó cinco complejos fenotípicos: Leishmania braziliensis, Leishmania naiffi, Leishmania guyanensis/Leishmania panamensis/ Leishmania shawi, Leishmania mexicana, Leishmania major para las poblaciones aisladas del Nuevo Mundo (4).

\footnotetext{
Correspondencia:

Clemencia Elena Ovalle, Av. 1 \# 13A-61 Bogotá, Colombia Teléfono: (571) 2428160, ext. 115,145,137; fax: (571) 3373597

investigacion@dermatologia.gov.co
}

Recibido: 29/07/05; aceptado: 06/03/06
En Colombia, la leishmaniasis es una enfermedad endémica en casi todo el territorio con excepción de San Andrés, Atlántico y Bogotá, D.C. (5), constituyéndose en un problema de salud pública. En el año 2004 se notificaron 10.794 casos, de los cuales, 98,4\% (10.624) correspondió a leishmaniasis cutánea, $1 \%(96)$ a leishmaniasis visceral y el $0,6 \%$ (74) restante a leishmaniasis mucosa. En este periodo los departamentos que reportaron mayor incidencia de leishmaniasis cutánea fueron, en su orden: Tolima, Antioquia, Santander, Norte de Santander, Caquetá, Nariño, Cundinamarca, Boyacá, Guaviare, Caldas y Risaralda (6).

Desde hace 20 años se han realizado esfuerzos para determinar la distribución geográfica de las especies de Leishmania en el país; es así como en 1986 se publicó el primer informe de distribución geográfica de 225 aislamientos (7) y en 1990 se reportaron los resultados de un estudio de 340 aislamientos de humanos, animales y vectores de varias regiones de Colombia, donde predominó la $L$. panamensis $(53,8 \%), L$. braziliensis $(30,3 \%)$, L. chagasi $(9,4 \%), L$. guyanenesis $(2,6 \%)$, L. amazonensis $(1,8 \%), L$. mexicana $(0,9 \%)$ y Leishmania sp. (1,2\%) (5).

En 1998 se identificaron 511 aislamientos de pacientes consultantes al Centro Internacional de Investigaciones Médicas (CIDEIM), donde encontraron que $L$. braziliensis estaba distribuida en varias zonas del país, $L$. panamensis era prevalente en el occidente colombiano, y $L$. guyanensis en la riberas de los ríos Orinoco y Amazonas (8).

El Instituto Nacional de Dermatología atiende pacientes provenientes de todo el país, con diferentes formas clínicas de leishmaniasis, a 
quienes se les aísla y caracteriza el agente etiológico. Este estudio pretende describir la distribución de las especies de Leishmania en Colombia aisladas a partir de los pacientes consultantes al Instituto Nacional de Dermatología durante el período de 1995 a 2005.

\section{Materiales y métodos}

Tipo de estudio. Se realizó un estudio descriptivo de la distribución geográfica de las especies de Leishmania.

Población de estudio. Se incluyeron los aislamientos obtenidos de 137 pacientes atendidos por dermatólogos en la consulta especial de leishmaniasis, los cuales presentaban diagnóstico confirmado de leishmaniasis cutánea, difusa, cutánea y mucosa simultánea; se revisó la historia clínica y se obtuvo información relacionada con forma clínica, sitio probable de infección, edad y sexo.

Obtención de la muestra. La muestra se obtuvo por punción aspirativa del borde de las lesiones cutáneas que se caracterizaban por múltiples placas ulceradas con bordes violáceos infiltrados de diferentes tamaños, localizadas en varios sitios anatómicos.

Aislamiento y cultivo. El aspirado se colocó en en medio de Seneckjie, se incubó a $26^{\circ} \mathrm{C}$ y se observó durante cinco semanas (9). Los parásitos aislados se cultivaron en medio de Schneider Drosophila (Sigma Chemical Co.) con suplemento de suero fetal bovino al 10\%, inactivado, para obtener cultivo en masa. Una vez que el cultivo de parásitos se encontraba en fase logarítmica de crecimiento se ajustó la población a $3 \times 10^{6}$ parásitos por $\mathrm{ml}$ con el fin de preparar el antígeno de acuerdo con el protocolo de la Organización Panamericana de la Salud (OPS) (10).

Tipificación de los aislamientos. La tipificación de las especies se hizo mediante anticuerpos monoclonales (10). Se emplearon 11 anticuerpos monoclonales (cuadro 1), los cuales fueron producidos por McMahon Pratt y John R. David 1981 (11) y donados por el CIDEIM.

Los patrones obtenidos para la identificación de especies se determinaron por la posibilidad de los anticuerpos monoclonales, así: L. braziliensis con B-18, B-16, B-2 y B-21; L. panamensis con B-4, B-11, B-2 y B-21; L. guyanensis con B-19 y B-2; complejo L. mexicana con M-2 y M-7; L. mexicana con $\mathrm{M}-2$, M-7 y M-8.

Se utilizaron como controles las cepas de referencia: $L$. braziliensis MHOM/BR/75/M2903, L. panamensis MHOM/PA/71/LS-94, L. guyanensis MHOM/BR/75/M4147, L. mexicana MHOM/BZ/ 82/BEL21, L. amazonensis IFLA/BR/67/PH80, donadas por el CIDEIM.

Determinación de serodemas. Se analizaron los patrones de reactividad que presentaron los aislamientos con los anticuerpos monoclonales utilizados.

Comparación de la tipificación de especies por otros métodos. Se tomó el $10 \%$ de los aislamientos en forma ciega y se enviaron al laboratorio de referencia para ser caracterizados mediante patrones de isoenzimas y poder establecer la concordancia entre especies y

Cuadro 1. Descripción de anticuerpos monoclonales

\begin{tabular}{llc}
\hline Especificidad & Clon del hibridoma & Código del anticuerpo \\
\hline L. braziliensis/L. panamensis & VI-6H9 & $\mathrm{B}-21$ \\
L. braziliensis (todas las especies) & VI-4B9-D10 & $\mathrm{B}-2$ \\
L. braziliensis & XIII-3E6-B11 & $\mathrm{B}-16$ \\
L. braziliensis & $\mathrm{XIV}-2 \mathrm{~A} 5-\mathrm{A} 10$ & $\mathrm{~B}-18$ \\
L. panamensis & VI-2A5-A4 & $\mathrm{B}-4$ \\
L. panamensis & VII-5G3-F3 & $\mathrm{B}-11$ \\
L. guyanensis & $\mathrm{XLIV}-5 \mathrm{~A} 2-\mathrm{B} 9-\mathrm{B} 6$ & $\mathrm{~B}-19$ \\
L. mexicana/L. amazonensis & LXVIII-1D7-D8 & $\mathrm{M}-7$ \\
L. mexicana/L. amazonensis & - & $\mathrm{M}-2$ \\
L. amazonensis & IX-5H9-C10 & $\mathrm{M}-3$ \\
L. mexicana & IXVIII-4D8-E3 & $\mathrm{M}-8$ \\
\hline
\end{tabular}


complejos con los resultados obtenidos con anticuerpos monoclonales.

Así mismo, se tipificaron por PCR, utilizando dos pares de iniciadores, B1/B2 (12) y M1/M2 (13) específicos para el complejo L. braziliensis y complejo L. mexicana, respectivamente.

Consideraciones éticas. A todos los pacientes atendidos en la consulta especial de leishmaniasis del Centro Dermatológico Federico Lleras Acosta se les informa e indaga sobre la inclusión de los aislamientos (en el caso de que fuere posible) en el banco biológico institucional para posteriores investigaciones. El protocolo del presente estudio fue sometido al Comité de Ética institucional que lo categorizó como investigación sin riesgo, según la declaración de Helsinki.

\section{Resultados}

La frecuencia de las especies encontradas en los 137 aislamientos estudiados fueron: L. panamensis, 74,45\%; L. braziliensis, 15,33\%; L. guyanensis, $0,73 \%$; L. mexicana, $5,11 \%$, complejo mexicana, 3,65\%, y Leishmania sp. 0,73\%. La distribución por departamento de probables sitio de infección se muestra en el cuadro 2 y en la figura 1.
La reactividad de los anticuerpos monoclonales M-2 y M-7 para la tipificación del complejo $L$. mexicana permitió hacer la identificación confiable de los aislamientos a diferencia de lo reportado por otros autores que presentan resultados contradictorios $(14,15)$. Los anticuerpos monoclonales $\mathrm{M}-3$ y $\mathrm{M}-8$, utilizados para la identificación de especies $L$. amazonensis y $L$. mexicana, respectivamente, no presentaron resultados consistentes, lo cual no permitió la tipificación de 12 aislamientos (8,76\%); ocho de éstos $(5,10 \%)$ se identificaron como L. mexicana por el método de perfil isoenzimático.

La forma clínica según la especie causante se observa en el cuadro 3; se encontró que la forma más frecuente era la leishmaniasis cutánea $(97,08 \%)$, seguida de la leishmaniasis cutánea y mucosa simultáneamente $(2,19 \%)$ y, finalmente, la leishmaniasis difusa $(0,73 \%)$.

La leishmaniasis cutánea fue causada por $L$. panamensis en $74,44 \%$, L. braziliensis en $15,79 \%$, L. guyanensis en $0,75 \%$, el complejo L. mexicana en $3,76 \%$, L. mexicana $4,51 \%$ y Leishmania sp. en $0,75 \%$. La leishmaniasis mucosa y cutánea

Cuadro 2. Distribución geográfica de Leishmania aisladas de pacientes consultantes al Instituto Nacional de Dermatología Federico Lleras Acosta, E. S. E, 1995-2005

\begin{tabular}{|c|c|c|c|c|c|c|c|}
\hline \multirow[b]{2}{*}{ Departamentos } & \multicolumn{6}{|c|}{ Especies de Leishmania } & \multirow[b]{2}{*}{ Total } \\
\hline & $\begin{array}{c}L . \\
\text { braziliensis }\end{array}$ & $\begin{array}{c}L . \\
\text { panamensis }\end{array}$ & $\begin{array}{c}L . \\
\text { guyanensis }\end{array}$ & $\begin{array}{c}L . \\
\text { mexicana }\end{array}$ & $\begin{array}{l}\text { Complejo } \\
\text { L. mexicana }\end{array}$ & $\begin{array}{c}\text { Leishmania } \\
\text { sp. }\end{array}$ & \\
\hline Antioquia & 0 & 3 & 0 & 0 & 0 & 0 & 3 \\
\hline Bolívar & 0 & 2 & 0 & 0 & 0 & 0 & 2 \\
\hline Boyacá & 0 & 14 & 0 & 0 & 0 & 0 & 14 \\
\hline Caldas & 0 & 7 & 0 & 1 & 0 & 0 & 8 \\
\hline Caquetá & 0 & 1 & 0 & 1 & 0 & 0 & 2 \\
\hline Casanare & 0 & 0 & 0 & 2 & 1 & 0 & 3 \\
\hline Cauca & 0 & 0 & 0 & 1 & 0 & 0 & 1 \\
\hline Chocó & 0 & 3 & 0 & 0 & 0 & 0 & 3 \\
\hline Córdoba & 0 & 1 & 0 & 0 & 0 & 0 & 1 \\
\hline Cundinamarca & 15 & 15 & 0 & 1 & 2 & 0 & 33 \\
\hline Guaviare & 1 & 2 & 1 & 0 & 1 & 0 & 5 \\
\hline Huila & 0 & 1 & 0 & 0 & 0 & 0 & 1 \\
\hline Meta & 2 & 3 & 0 & 0 & 0 & 1 & 6 \\
\hline Risaralda & 0 & 1 & 0 & 0 & 0 & 0 & 1 \\
\hline Santander & 0 & 40 & 0 & 0 & 1 & 0 & 41 \\
\hline Tolima & 0 & 9 & 0 & 0 & 0 & 0 & 9 \\
\hline Valle del Cauca & 0 & 0 & 0 & 1 & 0 & 0 & 1 \\
\hline Vichada & 3 & 0 & 0 & 0 & 0 & 0 & 3 \\
\hline Total & 21 & 102 & 1 & 7 & 5 & 1 & 137 \\
\hline
\end{tabular}



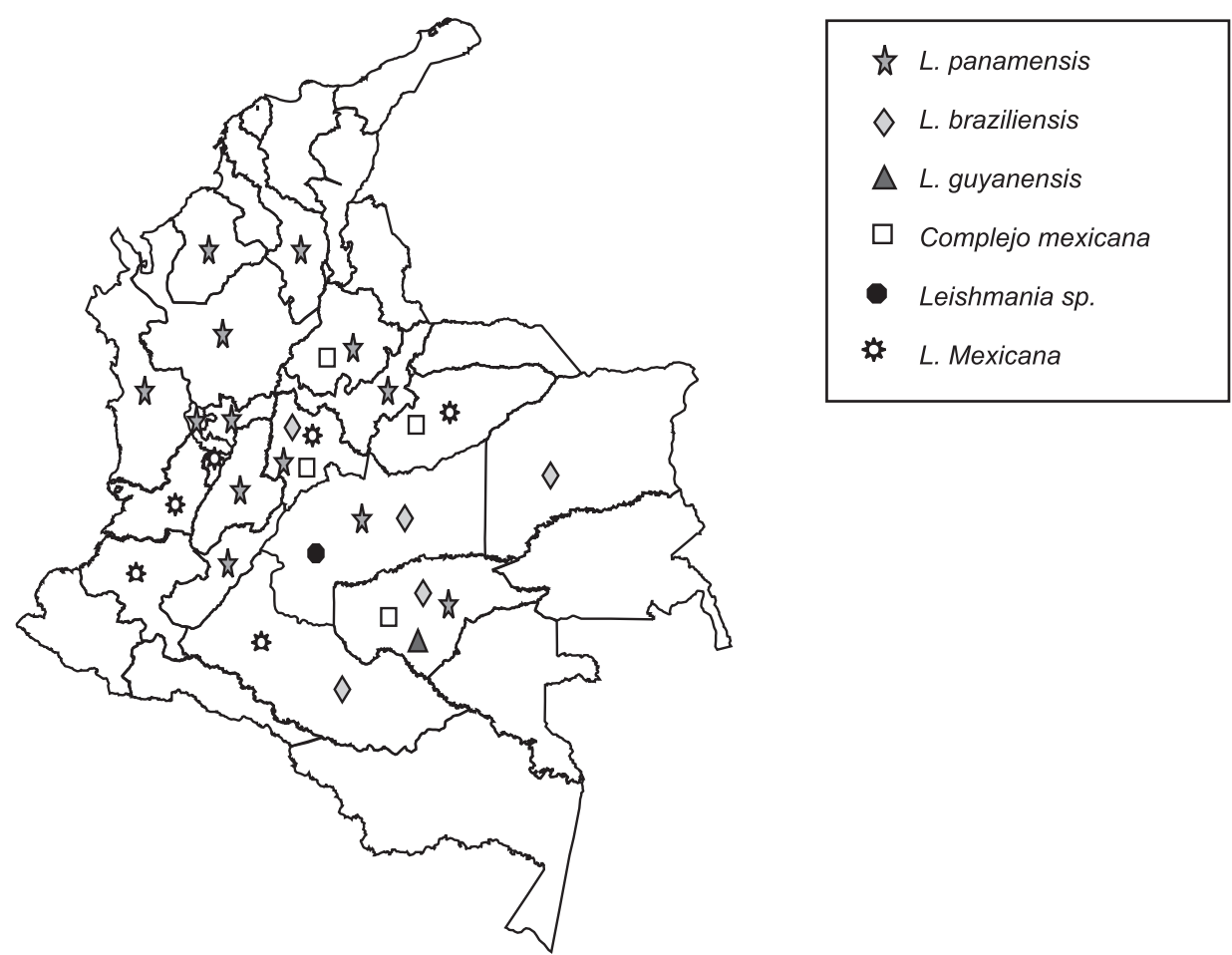

Figura 1. Distribución geográfica de especies de Leishmania aisladas de consultantes del Instituto Nacional de Dermatología, 1995-2005.

simultánea fue causada por $L$. panamensis en $66,67 \%$ y por L. mexicana $33,33 \%$. El caso de leishmaniasis difusa fue de $L$. panamensis, de un paciente inmunocomprometido con sida.

En cuanto al perfil de isoenzimas realizado en el laboratorio de referencia se pudo caracterizar el $93 \%$ de los complejos (13 aislamientos); los resultados presentaron $100 \%$ de concordancia con los obtenidos por anticuerpos monoclonales y PCR; estas dos últimas técnicas permitieron identificar el $100 \%$ de los complejos.

En lo relacionado con la identificación de especies, el perfil de isoenzimas permitió caracterizar el $93 \%$ (13 aislamientos) y los anticuerpos monoclonales el 42,8\% (6 aislamientos); por esta última técnica no fue posible identificar ninguna especie perteneciente al complejo L. mexicana. Una caracterización de aislamiento no coincidió entre las dos técnicas; fue caracterizado como $L$. panamensis por anticuerpos monoclonales y como L. braziliensis por perfil de isoenzimas (cuadro 4).
Cuadro 3. Presentación de la forma clínica de los casos de leishmaniasis

\begin{tabular}{lrccr}
\hline \multirow{2}{*}{ Especies } & \multicolumn{4}{c}{ Forma clínica* } \\
\cline { 2 - 5 } & LC & LC-LM & LD & Total \\
\hline L. brazilienzis & 21 & 0 & 0 & 21 \\
L. panamensis & 99 & 2 & 1 & 102 \\
L. guyanensis & 1 & 0 & 0 & 1 \\
L. mexicana & 6 & 1 & 0 & 7 \\
Complejo L. mexicana & 5 & 0 & 0 & 5 \\
Leishmania sp. & 1 & 0 & 0 & 1 \\
Total & $\mathbf{1 3 3}$ & $\mathbf{3}$ & $\mathbf{1}$ & $\mathbf{1 3 7}$ \\
\hline
\end{tabular}

*LC: leishmaniasis cutánea; LC-LM: leishmaniasis cutánea y leishmaniasis mucosa simultánea; LD: leishmaniasis difusa.

\section{Discusión}

A pesar de que el presente estudio corresponde a la casuística del Instituto Nacional de Dermatología Federico Lleras Acosta, lo cual introduce un sesgo de selección, los resultados obtenidos en cuanto a distribución de las especies de L. braziliensis, L.panamensis y L. guyanensis 
Cuadro 4. Resultados de la comparación de métodos para la identificación de aislamientos de Leishmania sp.

\begin{tabular}{llll}
\hline & \multicolumn{2}{c}{ Métodos de tipificación } & \\
\cline { 2 - 3 } Código de aislamiento & Anticuerpos monoclonales ${ }^{(1)}$ & Isoenzimas & \\
MHOM/CO/99/LL0002 & Complejo L. mexicana & L. mexicana & Complejo L. mexicana \\
MHOM/CO/99/LL0003 & Complejo L. mexicana & L. mexicana & Complejo L. mexicana \\
MHOM/CO/99/LL0004 & Complejo L. mexicana & L. mexicana & Complejo L. mexicana \\
MHOM/CO/99/LL0005 & Complejo L. mexicana & L. mexicana & Complejo L. mexicana \\
MHOM/CO/99/LL0006 & Complejo L. mexicana & L. mexicana & Complejo L. mexicana \\
MHOM/CO/00/LL0007 & Complejo L. mexicana & L. mexicana & Complejo L. mexicana \\
MHOM/CO/00/LL0008 & Complejo L. mexicana & ND & Complejo L. mexicana \\
MHOM/CO/00/LL0009 & Complejo L. mexicana & L. mexicana & Complejo L. mexicana \\
MHOM/CO/00/LL0010 & L. brazilienzis & L. braziliensis & Complejo L. braziliensis \\
MHOM/CO/00/LL0014 & L. brazilienzis & L. braziliensis & Complejo L. braziliensis \\
MHOM/CO/01/LL0019 & L. panamensis & L. panamensis & Complejo L. braziliensis \\
MHOM/CO/01/LL0023 & L. panamensis & L. panamensis & Complejo L. braziliensis \\
MHOM/CO/01/LL0024 & L. panamensis & L. braziliensis & Complejo L. braziliensis \\
MHOM/CO/01/LL0029 & L. panamensis & L. panamensis & Complejo L. braziliensis \\
\hline
\end{tabular}

(1) Instituto Nacional de Dermatología Federico Lleras Acosta, E.S.E., Laboratorio de Parasitología y Biología Molecular (2) CIDEIM, Unidad de Bioquimica y Biología Molecular

ND: No determinado

concuerdan con los obtenidos en estudios de coberturas más amplias como son los realizados por Corredor et al., Saravia et al. y Grimaldi et al. De igual forma, la especie más frecuentemente aislada es $L$. panamensis seguida por $L$. braziliensis $(5,7,16,17)$.

Por el contrario, la distribución de las especies del complejo L. mexicana mostró procedencias no reportadas previamente: La Victoria (Caldas), Barrancabermeja (Santander), La Mesa, Nimaima y Villeta (Cundinamarca), La Unilla (Guaviare), San José de Fragua (Caquetá), Tauramena, Ipigua y Monterrey (Casanare), municipio de Bolívar (Cauca) y Buenaventura (Valle). En estudios anteriores las especies de $L$. mexicana habían sido identificadas en los departamentos del Cauca, Meta, Nariño, Risaralda y Norte de Santander $(5,7,17)$; y la especie $L$. amazonensis se había aislado en el Meta y Norte de Santander $(5,17)$.

Los aislamientos identificados como L. panamensis presentaron un único patrón de serodema, lo cual coincide con el trabajo realizado por el CIDEIM en el 2002 (18). Igualmente, L. braziliensis también presentó un patrón único de serodema y en los trabajos de otros autores esta especie ha presentado hasta nueve patrones $(18,19)$. Estos hallazgos nos hacen suponer que el patrón de serodema no se encuentra relacionado con la distribución geográfica de las especies.
En cuanto a la tipificación del complejo $L$. mexicana consideramos que fue limitada la utilidad de los anticuerpos monoclonales M3 y M8 para la identificación de las especies $L$. amazonenesis y L. mexicana, respectivamente.

Dados los hallazgos de este estudio en el que se encontraron aislamientos de $L$. mexicana en las zonas geográficas en donde no se había reportado su presencia hasta ahora, apoya la hipótesis del origen antroponótico de la infección por los desplazamientos y la movilidad de las poblaciones. Así mismo, este hecho sugiere adelantar estudios de vectores con el fin de dilucidar este evento.

Con los resultados obtenidos en la caracterización se confirma que los patrones isoenzimáticos continúan siendo de gran utilidad en la identificación de especies; la especie que no se pudo identificar por isoenzimas se debió a la presencia de patrones que no coincidían con los presentados en las cepas de referencia y la baja reactividad del aislamiento; esto podría ser por la presencia de una variación dentro de la especie que se necesita comprobar con estudios adicionales.

\section{Agradecimientos}

Al CIDEIM por la donación de los anticuerpos monoclonales y cepas de referencia. A Rafael 
Góngora por la identificación de especies por el perfil de isoenzimas. Al Instituto Nacional de Salud por la donación de un anticuerpo monoclonal. A Sandra Moreno por su dedicación en el mantenimiento de cepas y preparación de antígeno.

\section{Conflicto de intereses}

Los autores manifiestan que no existe conflicto de intereses.

\section{Financiación}

El presente trabajo se realizó con la financiación del Instituto Nacional de Dermatología Federico Lleras Acosta, E.S.E.; recursos propios, código 20 del presupuesto de inversión.

\section{Referencias}

1. Sandoval CM, Angulo VM, Gutiérrez R, Muñoz G, Ferro C. Especies de Lutzomyia (Diptera: Psychodidae) posibles vectores de leishmaniasis en la ciudad de Bucaramanga, Santander, Colombia. Biomédica 1998;18:161-8.

2. Arias J, Beltrán F, Desjeux P, Walton B. Epidemiología y control de la leishmaniasis en las Américas, por país o territorio. Cuaderno técnico No. 44. Washington, D.C.: Organización Panamericana de la Salud; 1996. p.10-1.

3. Grimaldi G, Tesh RB. Leishmaniasis of the New World: current concepts and implication for future research. Clin Microbiol Rev 1993;6:230-50.

4. Cupolillo E, Momen H, Grimaldi G Jr. Genetic diversity in natural populations of New World Leishmania. Mem Inst Oswaldo Cruz 1998;93:663-8.

5. Corredor A, Kreutzer RD, Tesh RB, Boshell J, Palau MT, Cáceres E et al. Distribution and etiology of leishmaniasis in Colombia. Am J Trop Med Hyg 1990;42:20614.

6. Vera M, Galindo F, Zambrano P, Méndez J, Bello B, Olano V.Informe de enfermedades trasmitidas por vectores (ETV), 2004. Inf Quinc Epidemiol Nac 2005; $10: 34-48$.

7. Corredor A, Rey M, Hernández CA, Hernández LM, Parra MT. Leishmaniasis tegumentaria americana. Boletín Epidemiológico Nacional 1986;12:1-31.

8. Saravia NG, Segura I, Holguín AF, Santrich C, Valderrama L, Ocampo C. Epidemiologic, genetic and clinical associations among phenotypically distinct populations of Leishmania (Viannia) in Colombia. Am J Trop Med Hyg 1998;9:86-94.
9. Miranda MC, Posso CJ, Rojas C. Manual de normas y procedimientos para la atención de la leishmaniasis en los municipios del Valle del Cauca. Cali: Centro Internacional de Entrenamiento e Investigaciones Médicas; 1997. p.1-52.

10. Organización Mundial de la Salud. Uso de anticuerpos monoclonales en la identificación de Leishmania de importancia médica en Latinoamérica. Manual de procedimientos, Programa Especial para Investigación y Entrenamiento en Enfermedades Tropicales. Ginebra: Organización Mundial de la Salud; 1993. p.1-20.

11. McMahon-Pratt D, David JR. Monoclonal antibodies that distinguish between New World species of Leishmania. Nature 1981;291:581-93.

12. De Bruijn MHL, Barker DC. Diagnosis of the New World leishmaniasis: specific detection of species of the Leishmania braziliensis complex by amplification of kinetoplast DNA. Acta Trop 1992;52:145-58.

13. Eresh S, McCallum S, Barker DC. Identification and diagnosis of Leishmania mexicana complex isolates by polimerase chain reaction. Parasitology 1994;109:42333.

14. McMahon-Pratt D, Jaffe CL, Bennett E, David JR, Grimaldi G Jr. Studies employing monoclonal antibodies for the analysis of the genus Leishmania Ross 1903. En: Coll Intern CNRS/INSERM. Leishmania, taxonomie et phylogenese. Applications ecoepidemiologiques. Montpellier: IMEEE; 1986. p.173-8.

15. McMahon-Pratt D, Bennett E, Grimaldi G, Jaffe CL. Subspecies and species-specific antigens of Leishmania mexicana characterized by monoclonal antibodies. J Immunol 1985;134:1935-40.

16. Weigle KA, Saravia NG, de Davalos M, Moreno L, D'Alessandro A. Leishmania braziliensis from the Pacific Coast region of Colombia: foci of transmission, clinical spectrum and isoenzyme phenotypes. Am J Trop Med Hyg 1986;35:722-31.

17. Grimaldi G Jr, Tesh RB, McMahon-Pratt D. A review of the geographic distribution and epidemiology of leishmaniasis in the New World. Am J Trop Med Hyg 1989;41:687-725.

18. Saravia NG, Weigle K, Navas C, Segura I, Valderrama L, Valencia AZ et al. Heterogeneity geographic distribution and pathogenicity of serodemes of Leishmania viannia in Colombia. Am J Trop Med Hyg 2002;66:738-44

19. Grimaldi G Jr, David JR, McMahon-Pratt D. Identification and distribution of New World Leishmania species characterized by serodeme analysis using monoclonal antibodies. Am J Trop Med Hyg 1987;36:270-87. 